\title{
Classical Double-Layer Atoms: Artificial Molecules
}

\author{
B. Partoens, ${ }^{*}$ V. A. Schweigert, ${ }^{\dagger}$ and F. M. Peeters ${ }^{\ddagger}$ \\ Departement Natuurkunde, Universiteit Antwerpen (UIA), B-2610 Antwerpen, Belgium
}

(Received 30 June 1997)

\begin{abstract}
The ground-state configuration and the eigenmodes of two parallel two-dimensional classical atoms are obtained as a function of the interatomic distance $(d)$. The classical particles are confined by identical harmonic wells and repel each other through a Coulomb potential. As a function of $d$ we find several structural transitions which are of first or second order. For first (second) order transitions the first (second) derivative of the energy with respect to $d$ is discontinuous, the radial position of the particles changes discontinuously (continuously), and the frequency of the eigenmodes exhibits a jump (one mode becomes soft, i.e., its frequency becomes zero). [S0031-9007(97)04487-6]
\end{abstract}

PACS numbers: 73.20.Dx, 36.40.Ei, 64.90.+b

In recent years, there has been an increased interest in the study of finite two-dimensional (2D) systems of charged particles [1]. These quantum dots are atomiclike structures which have interesting optical properties and may be of interest for single electron devices. Most of the previous works have treated the quantum mechanical problem of a small number of electrons. If the number of electrons is increased beyond six or seven, the Coulomb correlation of the electrons has to be treated in an approximate way. Therefore, in order to fully understand the physics of Coulomb correlations, we have made a thorough study of the classical system [2] in which the particles are taken pointlike but where no approximation was made on the Coulomb correlation. This approach is valid in high magnetic fields, where the kinetic energy of the electrons is quenched. In the ground state the charged particles are located on rings, and a table of Mendeljev was constructed in Ref. [2]. These configurations were recovered in a quantum calculation in the limit of large magnetic fields [3]. A study of the spectral properties of these classical systems such as the energy spectrum, the eigenmodes, and the density of states was made in Ref. [4]. Such classical confined systems have been observed in electron dimples on liquid helium [5], in drops of colloidal suspensions [6], and in confined dusty particles [7].

Here we extend our previous work to the case of classical artificial molecules [8] which consist of two classical 2D atoms which are laterally separated by a distance $d$. Intuitively, we expect interesting behavior as a function of $d$ which governs the interatomic interaction. This can be seen as follows: For $d=0$ we have just one $2 \mathrm{D}$ atom (e.g., for $2 N=10$, this is a two ring structure), while for $d \rightarrow \infty$ the system consists of two independent 2D atoms with each half of the total number of particles (e.g., two atoms each having five particles on one ring). This implies that, as a function of $d$, structural transitions (e.g., configurational changes) have to take place. We found that these artificial molecules show a surprisingly complex behavior as a function of $d$. In this Letter, we investigate the ground-state energy and its derivatives, the ground-state configurations, and the normal modes, i.e., the eigenfrequencies, of the system.

We limit ourselves to the system consisting of an even number, $2 N$, of charged particles which are evenly distributed over two layers separated at a distance $d$. In both layers, the confinement potential is centered around the $z$ axis and this parabolic confinement, which is taken to be the same for both layers, keeps the system together in the $x y$ plane. We focus our attention on systems described by the Hamiltonian (where $m$ is the mass of the particles, $\omega_{0}$ the radial confinement frequency, $e$ the particle charge, and $\epsilon$ the dielectric constant of the medium that the particles are moving in),

$$
H=H_{\mathrm{I}}+H_{\mathrm{II}}+H_{\mathrm{I}, \mathrm{II}},
$$

with

$$
H_{S}=\sum_{i \in S} \frac{1}{2} m \omega_{o}^{2} r_{i}^{2}+\frac{e^{2}}{\epsilon} \sum_{i<j \in S} \frac{1}{\left|\vec{r}_{i}-\vec{r}_{j}\right|},
$$

the confinement and interaction energy of the artificial atom in layer $S=\mathrm{I}$, II and

$$
H_{\mathrm{I}, \mathrm{II}}=\frac{e^{2}}{\epsilon} \sum_{i \in \mathrm{I}} \sum_{j \in \mathrm{II}} \frac{1}{\left|\vec{r}_{i}-\vec{r}_{j}\right|},
$$

the interaction energy between the atoms in the two layers. For convenience, we will refer to our charged particles as electrons, keeping in mind that they can also be ions with charge $e$ and mass $m$. The Hamiltonian can be written in a dimensionless form if we express the coordinates, energy, and frequency in the following units $r^{\prime}=\left(e^{2} / \epsilon\right)^{1 / 3} \alpha^{-1 / 3}, E^{\prime}=\left(e^{2} / \epsilon\right)^{2 / 3} \alpha^{1 / 3}, \omega^{\prime}=\omega_{0} / \sqrt{2}$, respectively, with $\alpha=m \omega_{0}^{2} / 2$. In dimensionless units the Hamiltonian becomes

$$
\begin{aligned}
H= & \sum_{i \in \mathrm{I}} r_{i}^{2}+\sum_{i<j \in \mathrm{I}} \frac{1}{\left|\vec{r}_{i}-\vec{r}_{j}\right|}+\sum_{i \in \mathrm{II}} r_{i}^{2} \\
& +\sum_{i<j \in \mathrm{II}} \frac{1}{\left|\vec{r}_{i}-\vec{r}_{j}\right|}+\sum_{i \in \mathrm{I}} \sum_{j \in \mathrm{II}} \frac{1}{\left|\vec{r}_{i}-\vec{r}_{j}\right|},
\end{aligned}
$$


and consequently the ground-state energy is only a function of the number of electrons $2 N$ and the distance $d$ between the layers.

The numerical method used in the present study to obtain the ground-state configuration is based on the Monte Carlo technique supplemented with Newton's method in order to increase the accuracy of the energy of the groundstate configuration. The latter technique is outlined and compared with the Monte Carlo technique in Ref. [4] and also yields the eigenfrequencies and the eigenmodes of the ground-state configuration.

Let us consider first the case of a molecule consisting of six electrons distributed over two atoms, each with three electrons. For $d=0$ this is a $2 \mathrm{D}$ atom with six electrons, of which we know the ground-state configuration [2], namely, (1,5): five electrons on a ring and one electron in the center of the ring. In the opposite limit, $d \rightarrow \infty$, we have two independent $2 \mathrm{D}$ atoms, each consisting of three electrons for which the ground-state configuration consists of one ring containing three electrons [2]. Thus, as function of $d$, we expect a structural transition.

Figure 1 shows the energy per electron of the ground state and its first derivative with respect to $d$. At $d=$ 0.35955 , the first derivative is discontinuous and a structural transition takes place. For $d<0.35955$, the groundstate configuration is $(1,2) /(0,3)$ : the configuration $(1,2)$ in one layer (indicated by the open dots in the insets of Fig. 1) and $(0,3)$ in the other layer (indicated by the solid dots). As viewed from above we have $(1,5)$, which is the configuration of one atom consisting of six electrons. This implies that the interlayer correlations are sufficiently strong to impose the one atom configuration to the $2 N=6$ electrons in the molecular structure. For $d>0.35955$ the configuration is twice $(0,3)$, which is the configuration of two independent atoms, each consisting of three electrons.

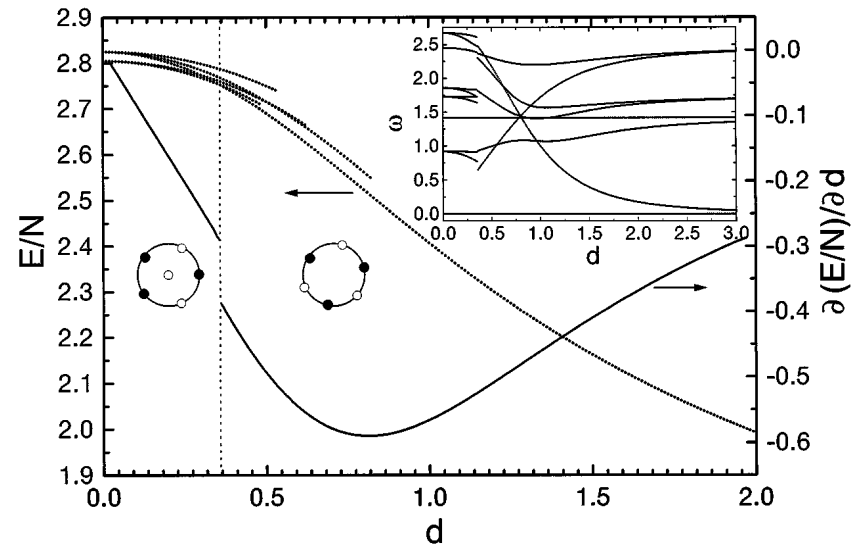

FIG. 1. The energy of the ground state (plotted as dots) and its first derivative with respect to the lateral distance $d$ between the two atoms constituting the artificial molecule for $2 \mathrm{~N}=6$. The inset shows the frequency of the normal modes of the ground state. The configurations are schematically represented in the figure, where solid and open dots correspond to electrons in different layers.
The frequencies of the normal modes of the groundstate configuration are shown in the inset of Fig. 1. Notice that at the first order transition point the frequencies exhibit a jump. For $d>0.35955$, more modes are degenerate in energy, and in the limit of $d \rightarrow \infty$ all eigenfrequencies are at least twofold degenerate. The latter is a consequence of the fact that the electrons in one layer can vibrate in phase and out of phase with respect to the electrons in the other layer. With decreasing $d$ the interlayer interaction destroys this degeneracy.

Also in Fig. 1, the energies of the metastable states are shown. For $d=0$ there is just one metastable state with configuration $(0,6)$. If $d$ is slightly different from zero, there are four metastable states plus the ground state. One obtains these configurations by moving one electron from one layer to the other without changing its lateral position, and this was done for each of the two configurations $[(1,5)$ and $(0,6)]$.

The artificial molecule consisting of $2 \times 5$ electrons is far more complex and exhibits several transitions, some of which are qualitatively different. For $d=0$ this is a $2 \mathrm{D}$ atom with ten electrons, and the groundstate configuration is $(2,8)$ [2]. For $d \rightarrow \infty$ we have two independent $2 \mathrm{D}$ atoms, both with configuration $(0,5)$ [2]. Figure 2(a) shows the eigenfrequencies as a function of $d$, and Fig. 2(b) shows the distance of the electrons from the center of the confinement potential. At first glance, we have four transition regions, namely, around $d=$ $0.16, d=0.68, d=0.78$, and $d=0.90$. The spatial configurations of the electrons are depicted in the inset of Fig. 2(b).

Region 1 (around $d=0.16$ ) and region 2 (around $d=0.68$ ). - The transitions in both regions are qualitatively similar, and therefore we limit our discussion to $d \approx 0.68$. At these transition points there is no abrupt change of the configuration, but within a small $d$ region the radii of the electrons change appreciably, but continuously [Fig. 3(a)]. Figure 3(b) shows that this region is delimited by two eigenfrequencies which become zero and, consequently, these transitions are induced by the softening of a mode. In Fig. 3(c) the second derivative of the energy with respect to $d$ is given. There are two discontinuities in the second derivative, namely, at $d=0.68120$ and $d=0.68215$ while the first derivative is continuous. In the inset of Fig. 3(c) the spatial configuration is shown at $d=0.681$ (circles) and at $d=0.683$ (triangles). The open and closed symbols refer to electrons belonging to different layers. Notice that no qualitative changes of the configuration occurs at the second order transitions. For region 1 we found similar second order transitions at $d=0.15775$ and $d=0.16175$.

Region 3 (around $d=0.78$ ). - The transition occurs at $d=0.77605$, where the position of the electrons changes discontinuously. There is a jump in the eigenfrequencies and in the first derivative of the energy. Clearly this is a first order transition which is similar to those found for the above molecule with $2 \times 3$ electrons. 

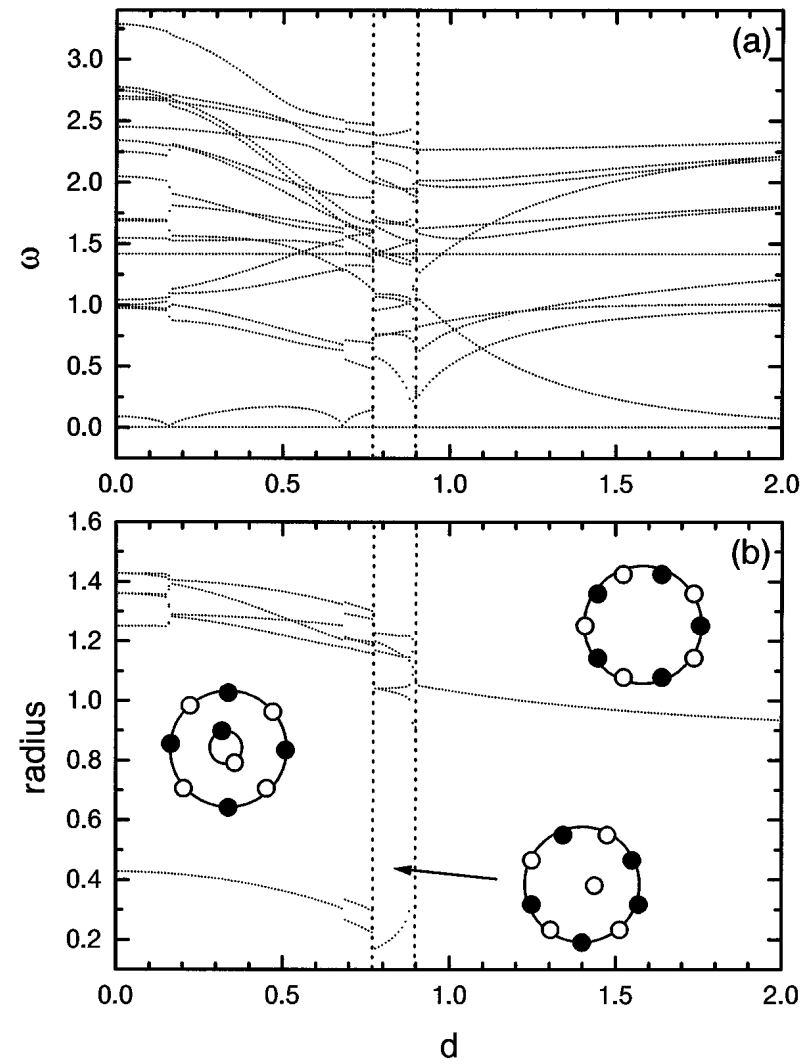

FIG. 2. (a) The eigenfrequencies of the ground state for the artificial molecule with $2 \times 5$ electrons as a function of the distance $d$ between the two atoms. (b) The distance of the different electrons from the center of the confinement potential. The three ground-state configurations are also shown in the insets of the figure.

Region 4 (around $d=0.90$ ). - - In this region there are two transitions: the first at $d=0.88585$ and the second at $d=0.90588$. Only in the latter does the number of electrons on the different rings change. It is a first order transition, and the eigenfrequencies exhibit a jump. On the other hand, the transition at $d=0.88585$ does not correspond to a change in the number of rings, however, there is a jump in the position of the electrons. It is a first order transition with a discontinuity in the frequency spectrum in which the smallest nonzero eigenfrequency decreases for $d \rightarrow 0.88585$ but stays different from zero.

For the artificial molecule in which each atom contains six electrons we find both first and second order transitions. With increasing $d$ there are three first order transitions, namely, at $d=0.2965, d=0.5125$, and $d=0.5345$, followed by one second order transition induced by the softening of a mode at $d=0.9259$. The latter is different from the previous molecule with $2 \times 5$ electrons where there are narrow $d$ regions delimited by two second order transitions. For $d<0.2965$ the configuration is $(2,4) /(1,5)$; at the first order transitions the position of the particles changes discontinuously while at the second order transition the position of the particles changes continuously and, after it, the outer elec-
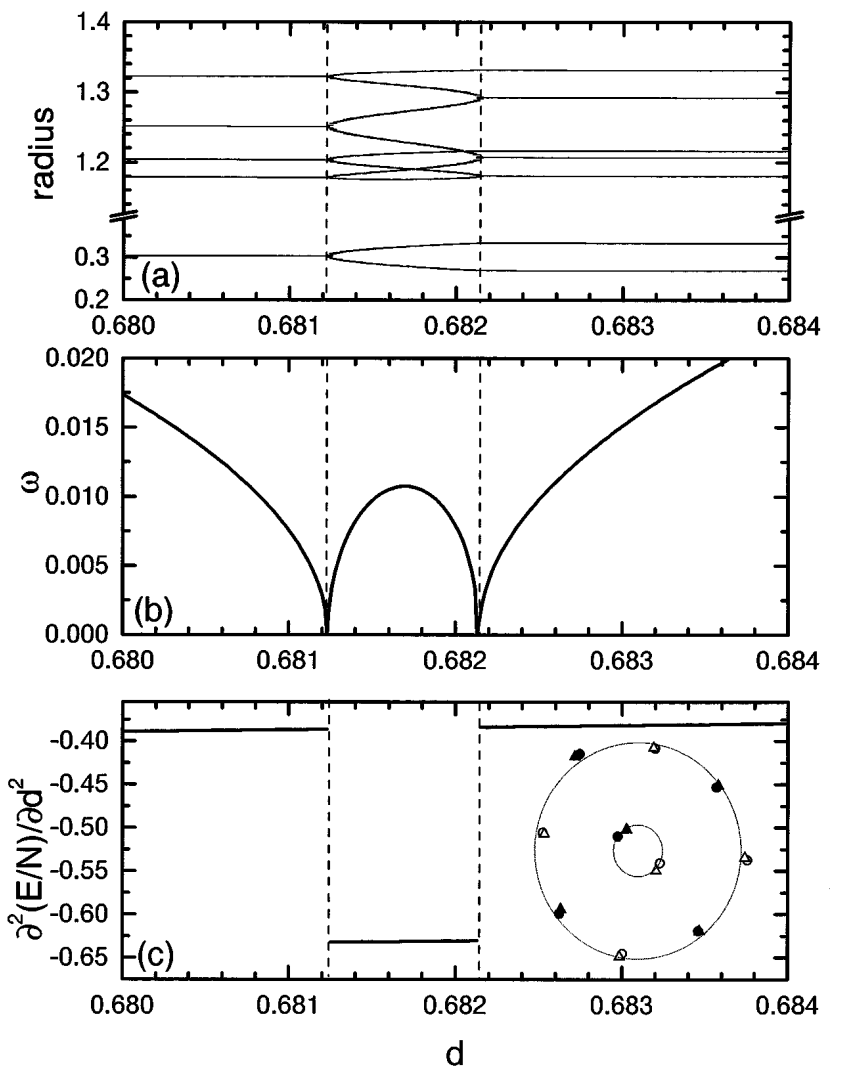

FIG. 3. (a) The distance of the electrons from the center of the confinement potential for the artificial molecule with $2 \times 5$ electrons as a function of $d$. (b) The lowest nonzero eigenfrequency of the ground state. (c) The second derivative of the ground-state energy with respect to $d$. The inset shows the configuration at $d=0.681$ (circles) and at $d=0.683$ (triangles). Open and solid symbols refer to electrons belonging to different atoms.

trons form a perfect circle with the inner electrons sitting in the center, resulting in the configuration $(1,5) /(1,5)$.

The ground state [2] of the artificial atom with seven electrons is $(1,6)$, and with eight electrons is $(1,7)$. Therefore, we expect that the above new type of transition also takes place for the artificial molecule with $2 \times 7$ and $2 \times 8$ electrons. For the molecule in which both atoms contain seven electrons, we find two narrow $d$ regions delimited by two second order transitions (the first at $d=$ 0.360375 and $d=0.360495$, the second at $d=0.504625$ and $d=0.504865$ ), a first order transition at $d=0.5425$, and indeed the second order transition at $d=0.9259$. Figure 4 shows the frequency spectrum and the position of the particles near the last transition. At the second order transition at $d=0.9259$ the system transforms from an imperfect ring structure, with the middle electron outside the center of the system, to a perfect ring with one electron in the center of each layer [see Fig. 4(b)].

For the latter molecule another phenomenon occurs. Around $d \approx 0.74$ the smallest nonzero eigenfrequency decreases substantially but does not become zero, while the other eigenfrequencies and the position of the particles change fast but continuously with $d$. It is clear that 

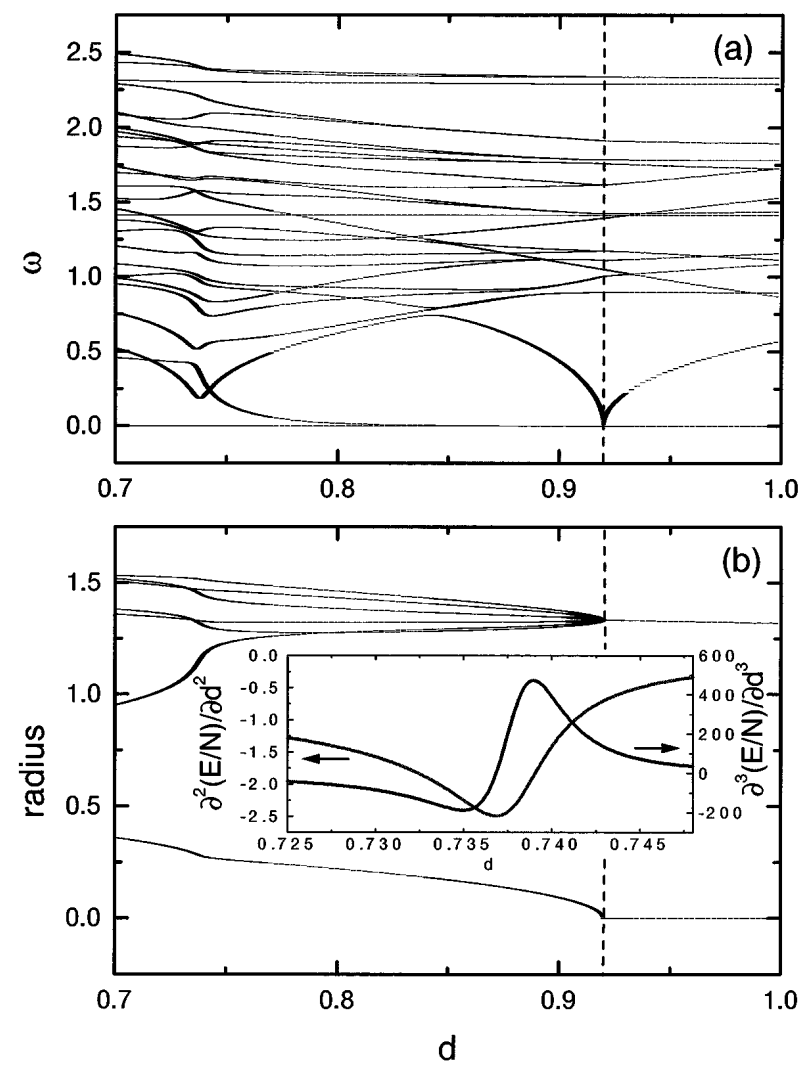

FIG. 4. (a) The eigenfrequencies of the ground state for the artificial molecule with $2 \times 7$ electrons as a function of $d$. (b) The distance of the electrons from the center of the confinement potential. In the inset, the second and third derivatives of the energy are shown at about $d \approx 0.74$.

this is not a first, second, or third order transition as is made clear in the inset of Fig. 4(b), where we plotted the second and third derivative of the energy with respect to $d$. Therefore we refer to this as a continuous transition with a lambdalike shape of the derivative $\partial^{3} E / \partial d^{3}$.

In conclusion, we have presented the results of a numerical calculation of the ground-state configuration, its energy and the spectrum of the normal modes of classical double-layer atoms. There is a vertical Coulomb coupling between the electrons constituting the molecule. These artificial molecules undergo structural transitions as a function of the distance between the layers. We found first and second order transitions. The different transitions are summarized in Fig. 5 in a $(d, N)$ diagram for molecules containing up to $2 N=20$ electrons. Although we assumed that (1) both atoms of the molecule contain the same number of electrons and (2) both atoms have the same confinement potential, a rich variety of structural transitions is found. It is expected that if we relax these assumptions new configurations are possible.

The present system constitutes a simple nontrivial model system exhibiting a rich variety of structural transitions. This finite system correctly describes the local properties of structural phase transitions. Furthermore, we found additionally: (1) transitions which are delimited by

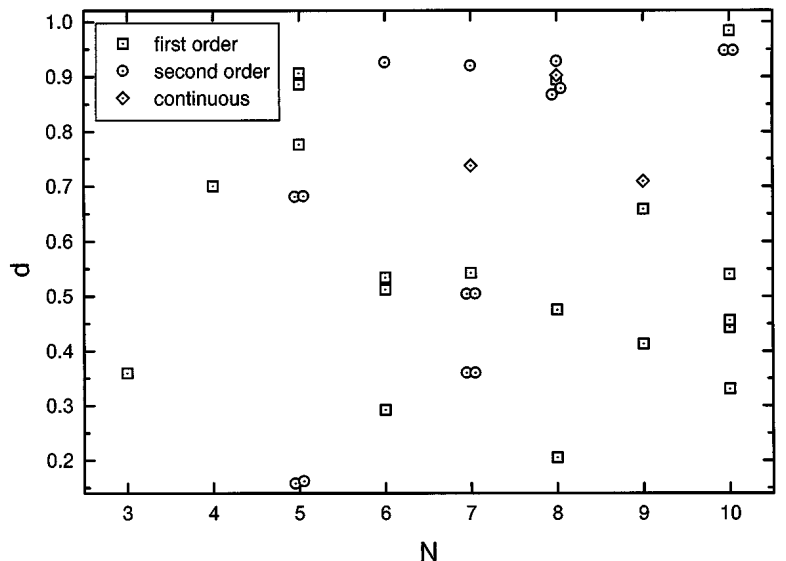

FIG. 5. Summary of the different transitions for molecules up to $2 N=20$ electrons. "Continuous" refers to a transition at which the position and the eigenfrequencies change rapidly, but continuously, with $d$, and the derivatives of the energy with respect to $d$ are continuous.

recurring second order transitions and (2) a lambda-type transition without a divergence in the second derivative of the energy. It should be noted that in the limit $N \rightarrow$ $\infty$ we obtain the system of two parallel classical twodimensional electron gases, each of which form a Wigner crystal which was studied in Ref. 9.

This work was supported by a "Krediet aan Navorsers" from the FWO-Vlaanderen, the Human Capital and Mobility network Programme No. ERBCHRXT 930374, INTAS-93-1495, and the Russian Foundation for Basic Research 96-02-19134. B.P. and F. M.P. are supported by FWO-VI.

*Electronic address: bpartoen@uia.ua.ac.be

${ }^{\dagger}$ Permanent address: Institute of Theoretical and Applied Mechanics, Russian Academy of Sciences, Novosibirsk 630090, Russia.

\#Electronic address: peeters@uia.ua.ac.be

[1] See, e.g., special issue on the Proceedings of the Workshop on Novel Physics in Low-Dimensional Electron Systems, edited by T. Chakraborty [Physica (Amsterdam) 212B, No. 3 (1995)].

[2] V. M. Bedanov and F. M. Peeters, Phys. Rev. B 49, 2667 (1994).

[3] P. A. Maksym, Physica (Amsterdam) 184B, 385 (1993).

[4] V.A. Schweigert and F. M. Peeters, Phys. Rev. B 51, 7700 (1995); F. M. Peeters, V. A. Schweigert, and V. M. Bedanov, Physica (Amsterdam) 212B, 237 (1995).

[5] P. Leiderer, W. Ebner, and V. B. Shikin, Surf. Sci. 113, 405 (1992).

[6] J.E. Hug, F. van Swol, and C.F. Zukoski, Langmuir 11, 111 (1995); S. Neser, C. Bechinger, P. Leiderer, and T. Palberg, Phys. Rev. Lett. 79, 2348 (1997).

[7] C. H. Chiang and L. I, Phys. Rev. Lett. 77, 647 (1996).

[8] R. Ugajin, Appl. Phys. Lett. 68, 2657 (1996); H. Imamura, P.A. Maksym, and H. Aoki, Phys. Rev. B 53, 12613 (1996).

[9] G. Goldoni and F. M. Peeters, Phys. Rev. B 53, 4591 (1996). 